\title{
Endothelin-Induced Sarcoplasmic Reticulum Calcium Depletion Waves in Vascular Smooth Muscle Cells
}

\author{
Mitra Esfandiarei, Yohan Choi, Arash Y. Tehrani, \\ Jeremy G. Hoskins, Nicola Fameli, Cornelis van Breemen \\ Child and Family Research Institute, \\ Department of Anesthesiology, Pharmacology, and Therapeutics, \\ The University of British Columbia, \\ Vancouver, British Columbia, V5Z 4H4, Canada
}

August 22, 2011

Agonist-stimulated waves of elevated cytoplasmic $\mathrm{Ca}^{2+}$ concentration $\left(\left[\mathrm{Ca}^{2+}\right]_{\mathrm{i}}\right)$ regulate blood vessel tone [1] and vasomotion [2] in vascular smooth muscle. Previous studies employing cytoplasmic $\mathrm{Ca}^{2+}$ indicators revealed that these $\mathrm{Ca}^{2+}$ waves were generated by a combination of inositol 1,4,5-trisphosphate $\left(\mathrm{IP}_{3}\right)$ and $\mathrm{Ca}^{2+}$ induced $\mathrm{Ca}^{2+}$ release (CICR) from the sarcoplasmic reticulum (SR) [3,4]; although, some of the mechanistic details remain uncertain. However, these findings were derived indirectly from observing agonist-induced $\left[\mathrm{Ca}^{2+}\right]_{\mathrm{i}}$ fluctuations in the cytoplasm.

Here, for the first time, we have recorded Endothelin-1 (ET-1) induced waves of $\mathrm{Ca}^{2+}$ depletion from the SR lumen in vascular smooth muscle cells (VSMCs) using a calsequestrin-targeted $\mathrm{Ca}^{2+}$ indicator. Our findings show that these waves: (1) are due to regenerative CICR by the receptors for $\operatorname{IP}_{3}\left(\mathrm{IP}_{3} \mathrm{R}\right),(2)$ have a marked latency period, (3) are characterized by a transient increase in SR $\mathrm{Ca}^{2+}\left(\left[\mathrm{Ca}^{2+}\right]_{\mathrm{SR}}\right)$ both at the point of origin and at the wave front, (4) proceed with diminishing velocity, and (5) are arrested by the nuclear envelope. Our quantitative model indicates that the gradual decrease in the velocity of the SR depletion wave, in the absence of external $\mathrm{Ca}^{2+}$, results from continuity of the SR luminal network.

In VSMCs, $\mathrm{Ca}^{2+}$ controls multiple functions, including contraction-relaxation, proliferation, migration and apoptosis, in health and disease [5-7]. This could be explained by the widely held view that functional selectivity of the $\mathrm{Ca}^{2+}$ signal is encoded in its spatial and temporal characteristics [8-10]. The first agonist-stimulated waves of elevated $\left[\mathrm{Ca}^{2+}\right]_{\mathrm{i}}$ in SMCs were reported in 1990 by Neylon et al [11], who proposed a mechanism of wave propagation based on interaction between $\mathrm{IP}_{3}$-sensitive receptors $\left(\mathrm{IP}_{3} \mathrm{Rs}\right)$ and ryanodinesensitive receptors (RyRs) located in separate endo/sarcoplasmic reticulum (ER/SR) com- 
partments [12]. With the discovery of excitatory $\mathrm{Ca}^{2+}$ sensors on $\mathrm{IP}_{3} \mathrm{R}$, a simpler model of regenerative CICR at the $\mathrm{IP}_{3}$ Rs was proposed $[10,13]$.

In order to obtain more direct evidence related to regenerative $\mathrm{SR} \mathrm{Ca}^{2+}$ release in VSMCs, we transfected cultured rat aortic SMCs with an adenoviral vector expressing a specific $\left[\mathrm{Ca}^{2+}\right]_{\mathrm{SR}}$ indicator that we refer to as D1SR. D1SR is a modified version of the $\mathrm{Ca}^{2+}$ sensitive cameleon, D1ER [14], in which the calreticulin signal sequence has been replaced by the calsequestrin signal sequence. The D1SR construct consists of enhanced CFP and YFP fluorescent proteins that are joined by a linker containing modified calmodulin (CaM) and M13 (the 26-residue CaM-binding peptide of myosin light-chain kinase) sequences [15]. Following binding to $\mathrm{Ca}^{2+}$, conformational changes in CaM-M13 domain increase the fluorescence resonance energy transfer (FRET) between the flanking CFP and YFP yielding a biphasic $\mathrm{Ca}^{2+}$ response. The specific targeting of the D1SR indicator to the SR lumen has provided us with a unique and valuable tool for monitoring real time changes in the $\left[\mathrm{Ca}^{2+}\right]_{\mathrm{SR}}$ in living SMCs.

Using fluorescence microscopy, we assessed the distribution of D1SR in our SMCs culture at $48 \mathrm{~h}$ post transfection. Figure 1a shows individual channels (CFP: $440 \mathrm{~nm} / 488 \mathrm{~nm}$; FRET: $440 \mathrm{~nm} / 535 \mathrm{~nm}$; YFP: $513 \mathrm{~nm} / 535 \mathrm{~nm}$ ), and the overlay of all three channels. The pattern of distribution of D1SR is similar to that of D1ER in another VSMC preparation and typical for smooth muscle SR [16]. As expected, D1SR also co-localizes with ER-Tracker except for a region close to the nucleus that is negative for D1SR fluorescence, but positive for ER-Tracker (Supplementary Figure 6a). Specific staining with Golgi-Tracker indicated that the Golgi apparatus is typically located in this region (Supplementary Figure 6b \& 6c).

Figure $1 \mathrm{~b}$ illustrates the effects of receptor activation with ET-1 (100 nM) and SERCA inhibition with $2 \mu \mathrm{M}$ thapsigargin $(\mathrm{Tg})$ on $\left[\mathrm{Ca}^{2+}\right]_{\mathrm{SR}}$ in the peripheral (gray line) and perinuclear (black dotted line) areas of SMCs. In the cell periphery, ET-1 causes a drop in $\left[\mathrm{Ca}^{2+}\right]_{\mathrm{SR}}$ and subsequent SERCA inhibition results in an additional drop. In the peri-nuclear areas, however, the ET-stimulated changes in $\left[\mathrm{Ca}^{2+}\right]_{\mathrm{SR}}$ are variable, and on average not significant. Although a clear loss of $\left[\mathrm{Ca}^{2+}\right]_{\mathrm{SR}}$ is evident following Tg application, a considerable amount of FRET/CFP signal remains, indicating the presence of organelles containing calsequestrin and $\mathrm{Ca}^{2+}$ that are not sensitive to SERCA blockade. A plausible explanation for this residual $\left[\mathrm{Ca}^{2+}\right]_{\mathrm{SR}}$ is that in cultured cells the $\mathrm{SR}$ is contiguous with membranous organelles or endosomes, which accumulate $\mathrm{Ca}^{2+}$ via the Tg-insensitive "secretory pathway $\mathrm{Ca}^{2+}$ ATPase" (SPCA). In support of this idea, we have found that, in the absence of external $\mathrm{Ca}^{2+}$, sodium orthovanadate $(1 \mathrm{mM})$, which inhibits all $\mathrm{Ca}^{2+}$ ATPases, abolishes the $\mathrm{Ca}^{2+}$ sensitive D1SR fluorescence (Supplementary figure 7).

In order to compare the above changes in $\left[\mathrm{Ca}^{2+}\right]_{\mathrm{SR}}$ with fluctuations in $\left[\mathrm{Ca}^{2+}\right]_{\mathrm{i}}$, we used the cytoplasmic $\mathrm{Ca}^{2+}$ indicator fluo-4AM in the same VSMC preparation (Fig. 1c). In these cells, ET-1 (100 nM) appears to be a weak agonist eliciting only a transient increase in $\left[\mathrm{Ca}^{2+}\right]_{\mathrm{i}}$ as opposed to uridine-5'-triphosphate (UTP), which stimulates a large maintained increase in $\left[\mathrm{Ca}^{2+}\right]_{\mathrm{i}}$ (data not shown). Subsequent inhibition of SERCA with $\mathrm{Tg}(2 \mu \mathrm{M})$ elicits another large $\left[\mathrm{Ca}^{2+}\right]_{\mathrm{i}}$ transient due to depletion of $\mathrm{SR}$ luminal $\mathrm{Ca}^{2+}$. Therefore, at least in the case of ET-1, it is quite clear that the luminal $\mathrm{Ca}^{2+}$ indicator shows different dynamics from those provided by the cytoplasmic $\mathrm{Ca}^{2+}$ sensitive dye, fluo-4AM.

To abolish the indirect effects of ET-1 stimulated $\mathrm{Ca}^{2+}$ entry from the extracellular space, we removed $\mathrm{Ca}^{2+}$ from the bathing solution. Under these conditions, which inhibit $\mathrm{Ca}^{2+}$ 


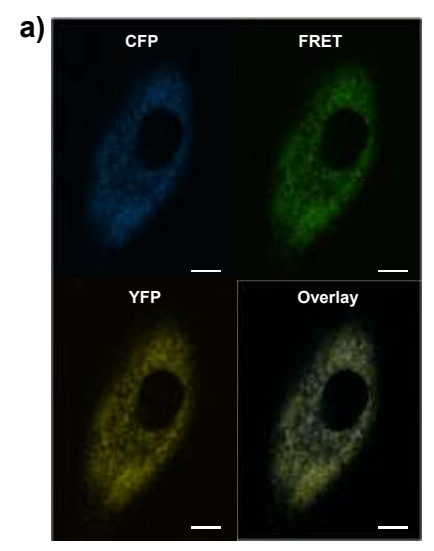

b)
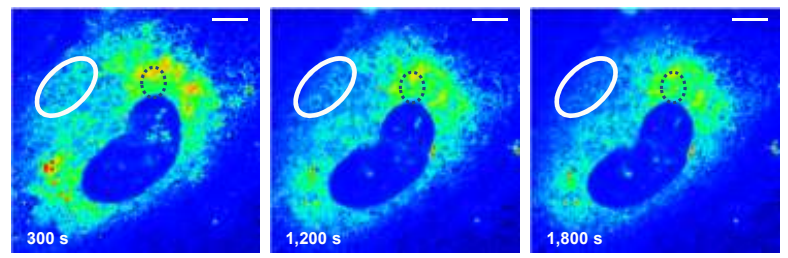

c)

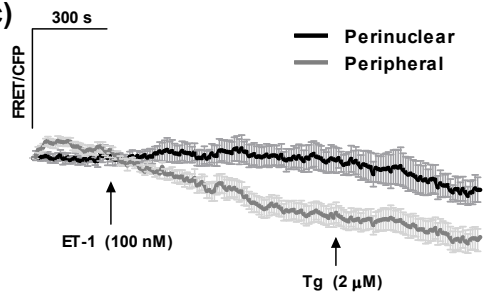

d)

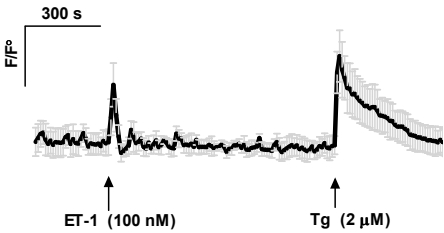

Figure 1: Effects of endothelin-1 and thapsigargin on SR and cytoplasmic $\mathrm{Ca}^{2+}$ signals in rat SMC culture. (a) Representative images of D1SR distribution in cultured rat SMCs. Fluorescence images of D1SR using CFP (440 nm excitation/488 nm emission), FRET (440 nm excitation/535 $\mathrm{nm}$ emission), and YFP (514 nm excitation/535 nm emission) band-pass filters and the overlay image of all three channels (scale bars, $10 \mu \mathrm{m}$ ). (b) Selected snapshots from a timelapse movie of rat SMCs transfected with D1SR indicator (scale bars, $5 \mu \mathrm{m}$ ), and corresponding FRET/CFP traces in peripheral (gray circle) and peri-nuclear (black dotted circle) areas in response to ET-1 $(100 \mathrm{mM})$ and $\mathrm{Tg}(2 \mu \mathrm{M})$ treatments. Fluorescence images were recorded with $10 \mathrm{~s}$ intervals for the duration of experiment. Traces represent overlay of averaged FRET/CFP signals in peripheral and peri-nuclear regions in the presence of extracellular calcium. ET-1 (100 mM) induces a steady and slow decrease in luminal $\mathrm{Ca}^{2+}$, while addition of $\mathrm{Tg}(2 \mu \mathrm{M})$ results in a slight further decrease. As illustrated, ET-1 does not cause any significant changes in signal intensity in the peri-nuclear area $(\mathrm{ROIs}=20, \mathrm{n}=5$ independent experiments, mean \pm s.e.m., $P>0.005$ for peri-nuclear areas, $P<0.005$ for peripheral areas). (c) Average trace of cytoplasmic $\mathrm{Ca}^{2+}$ signal in rat SMCs loaded with $\mathrm{Ca}^{2+}$ indicator Fluo-4AM. Fluorescence images (488 nm excitation/514 $\mathrm{nm}$ emission) were recorded with $10 \mathrm{~s}$ intervals for the duration of recording. ET-1 (100 nM) initially causes a spike in cytoplasmic $\mathrm{Ca}^{2+}$. Subsequent inhibition of SERCA with $2 \mu \mathrm{M}$ Tg elicits another large $\left[\mathrm{Ca}^{2+}\right]_{\mathrm{i}}$ transient due to depletion of $\mathrm{SR}$ luminal $\mathrm{Ca}^{2+}(\mathrm{ROI}=15, \mathrm{n}=4$ independent experiments, mean \pm s.e.m., $P<0.005)$.

refilling of smooth muscle SR [17], ET-1 caused a large, delayed drop in $\left[\mathrm{Ca}^{2+}\right]_{\mathrm{SR}}$ (Fig. 2a). In all recordings, a transient increase in $\left[\mathrm{Ca}^{2+}\right]_{\mathrm{SR}}$ is observed prior to its large rapid decline (marked by a black arrow). The clear difference in ET-induced responses in the presence and absence of external $\mathrm{Ca}^{2+}$ is not surprising, because under physiological conditions and in the presence of an external source of $\mathrm{Ca}^{2+}$, there is rapid $\mathrm{Ca}^{2+}$ cycling between the SR lumen and extracellular space [18], which appears to protect $\mathrm{SR}$ from $\mathrm{Ca}^{2+}$ depletion following ET-1 mediated stimulation. In contrast, in the absence of extracellular $\mathrm{Ca}^{2+}$, which inhibits SR refilling, ET-1 causes a much larger drop in $\left[\mathrm{Ca}^{2+}\right]_{\mathrm{SR}}$.

Furthermore, in about $80 \%$ of the cell populations, removal of external $\mathrm{Ca}^{2+}$ revealed a most interesting novel phenomenon that we refer to as the "SR $\mathrm{Ca}^{2+}$ depletion wave". Representative snapshots of a time-lapse confocal microscopy movie of a typical $\mathrm{Ca}^{2+}$ depletion wave are shown in figure $2 \mathrm{~b}$. At $300 \mathrm{~s}, \mathrm{Ca}^{2+}$ is removed from the bathing solution, and at 

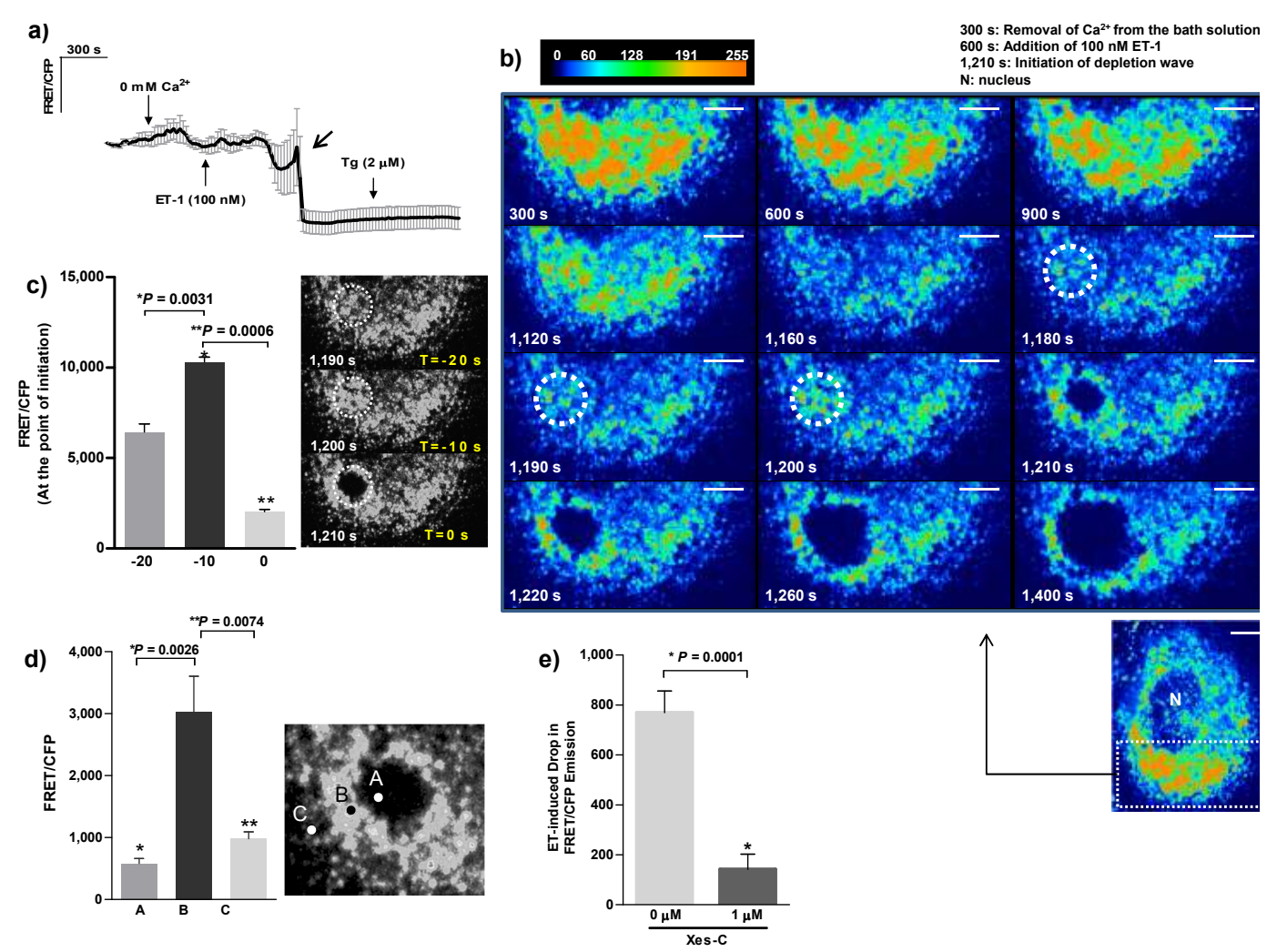

Figure 2: Progression of ET-induced regenerative $\mathrm{Ca}^{2+}$ depletion wave in the absence of extracellular $\mathrm{Ca}^{2+}$. (a) Average trace for FRET/CFP signal in SMCs treated with ET-1 $(100 \mathrm{nM})$ and $\mathrm{Tg}(2 \mu \mathrm{M})$ in the absence of extracellular $\mathrm{Ca}^{2+}$. ET-1 induces a delayed drop in the $\mathrm{Ca}^{2+}$, followed by a local transient increase in the recorded signal (black arrow). About 10 minutes post ET-1 addition, there is a significant drop in $\mathrm{Ca}^{2+}$ signal due to complete SR depletion $(\mathrm{n}=6$ independent experiments, mean \pm s.e.m., $P<0.005)$. (b) Snapshots (FRET channel) of time-lapse movie (10 s intervals) of SMCs illustrating ET-induced $\mathrm{Ca}^{2+}$ depletion wave (scale bars, $4.3 \mu \mathrm{m}$ ). Fluorescence images were recorded at $10 \mathrm{~s}$ intervals. Seconds refer to the time elapsed from the start of the recording. SR luminal $\mathrm{Ca}^{2+}$ level is indicated by the pseudo-colour intensity as marked by the scale. At $300 \mathrm{~s}$, the bathing solution is replaced by $0 \mathrm{mM} \mathrm{Ca}^{2+}$ HEPES. At $600 \mathrm{~s}, \mathrm{ET}-1$ $(100 \mathrm{nM})$ is added, eliciting a significant decrease in luminal calcium only 1,160 s post treatment. Immediately prior to wave initiation, a rapid transient increase in $\mathrm{Ca}^{2+}$ is observed at the point of origin (1,200 s panel, circled area), followed by a significant drop due to wave development in roughly circular fashion. (c) Average changes in $\mathrm{Ca}^{2+}$ signal intensity at the points of initiation. $\mathrm{T}=0 \mathrm{~s}$ marks the wave start, $\mathrm{T}=-10 \mathrm{~s}$, and $\mathrm{T}=-20 \mathrm{~s}$ refer to times prior to wave initiation. $\mathrm{Ca}^{2+}$ signal significantly increases before wave initiation $(\mathrm{T}=-10 \mathrm{~s})$, and then drops as the wave starts at $\mathrm{T}=0 \mathrm{~s}\left(\mathrm{n}=5\right.$ cells from five independent experiments, mean \pm s.e.m., $\left.{ }^{*} P=0.0031,{ }^{* *} P=0.0006\right)$. (d) FRET/CFP signal in three regions of the depletion wave (A: already depleted area; B: area that has up-taken the released $\mathrm{Ca}^{2+}$; $\mathrm{C}$ : area that will be up-taking $\mathrm{Ca}^{2+}$ released from area $\mathrm{B}$ ). At any given time point, the signal intensity at the rim (point B) is higher than both the already depleted area (point A) and the adjacent yet-to-be-refilled SR locus (point $\mathrm{C})(\mathrm{ROI}=4, \mathrm{n}=5$ cells from five independent experiments, mean \pm s.e.m., ${ }^{*} P=0.0026,{ }^{* *} P=0.0074$ ). (e) Inhibition of $\mathrm{IP}_{3} \mathrm{R}$ with Xes-C $(1 \mu \mathrm{M})$ blocks ET-induced depletion waves as compared to vehicle-control groups. For Xes-C treated groups, cells were perfused with $0 \mathrm{mM}$ HEPES $\mathrm{Ca}^{2+}$ buffer containing $1 \mu \mathrm{M}$ Xes-C starting $5 \mathrm{~m}$ prior to addition of ET-1 (0.1\% DMSO) ( $\mathrm{n}=5$ cells from five independent experiments, mean \pm s.e.m., ${ }^{*} P=0.0001$ ). 
$600 \mathrm{~s}, \mathrm{ET}-1(100 \mathrm{nM})$ is added. In the presence of ET-1, focal fluctuations in $\left[\mathrm{Ca}^{2+}\right]_{\mathrm{SR}}$ can be observed, and just before the onset of the $\mathrm{Ca}^{2+}$ depletion wave, $\left[\mathrm{Ca}^{2+}\right]_{\mathrm{SR}}$ is elevated at the site of wave initiation (Fig. 2b, panel 1,200 s). Measurements of changes in the FRET/CFP signal at the point of wave initiation from independent experiments confirm the transient increase in $\left[\mathrm{Ca}^{2+}\right]_{\mathrm{SR}}$ immediately prior to initiation of the depletion wave (Fig. 2c). Following this focal increase, complete depletion of $\left[\mathrm{Ca}^{2+}\right]_{\mathrm{SR}}$ occurs at the site of initiation, from which a wave of $\mathrm{Ca}^{2+}$ depletion radiates out into the surrounding SR network (Fig. 2b, panels $1,210-1,400 \mathrm{~s})$. The depletion wave progresses in a roughly circular fashion and is always surrounded by a rim of increased $\left[\mathrm{Ca}^{2+}\right]_{\mathrm{SR}}$. It thus appears that some of the $\mathrm{Ca}^{2+}$ released at the wave front are taken up by adjacent, not yet activated SR. This observation is validated by the data in figure $2 \mathrm{~d}$, which shows that, at any given time point of wave expansion, there are significant differences in $\left[\mathrm{Ca}^{2+}\right]_{\mathrm{SR}}$ between the depleted SR region (marked as A), the transiently refilled neighbouring SR locus (marked as B), and the next SR locus further ahead of the depletion wave (marked as C).

One plausible explanation for the initiation of $\mathrm{SR} \mathrm{Ca}^{2+}$ depletion waves is that after the $\mathrm{IP}_{3}$ concentration builds up to a critical level, and the cytoplasmic and $\mathrm{SR} \mathrm{Ca}^{2+}$ at the site of wave origin reach threshold, the $\mathrm{IP}_{3} \mathrm{Rs}$ on the SR membrane open in a regenerative fashion; the wave is then propagated by $\mathrm{CICR}$ at $\mathrm{IP}_{3} \mathrm{Rs}$. To test this hypothesis, we treated cultured SMCs with a specific $\mathrm{IP}_{3} \mathrm{R}$ inhibitor xestospongin $\mathrm{C}$ (Xes-C) prior to the application of ET1. Our results showed that Xes-C (1 M) completely blocked ET-induced depletion waves in cultured SMCs (Fig. 2e). The fact that the depletion wave is blocked by inhibition of $\mathrm{IP}_{3} \mathrm{R}$ indicates that like other vascular smooth muscle $\mathrm{Ca}^{2+}$ waves, the underlying mechanism is indeed mediated by $\mathrm{CICR}$ at $\mathrm{IP}_{3} \mathrm{R}[10,17,19]$. This notion was further corroborated by the observation that our cultured rat SMCs failed to respond to caffeine treatment, confirming the lack of functional RyRs in these cells (data not shown), which is in agreement with previous report by Vallot et al [20].

Another instructive way of analyzing the $\left[\mathrm{Ca}^{2+}\right]_{\mathrm{SR}}$ depletion wave is to identify a line intersecting the site of wave initiation and recording the changes in FRET/CFP signal along this line vs. time (line scan). The resulting "heat map" illustrates that initially the area of $\left[\mathrm{Ca}^{2+}\right]_{\mathrm{SR}}$ depletion expands rapidly, but as time proceeds, it asymptotically approaches a final limit (Fig. 3a). Analysis of a number of such heat maps yields the average distance vs. time, as well as the time course of the velocity of the depletion wave (Fig. 3b). Extrapolation of the velocity curve to the time of wave initiation $(0 \mathrm{~s})$, shows that the $\mathrm{SR} \mathrm{Ca}^{2+}$ depletion wave has an initial velocity of about $1 \mathrm{~m} / \mathrm{s}$, which falls below the range $(2-30 \mathrm{~m} / \mathrm{s})$ reported for intact smooth muscle $\mathrm{Ca}^{2+}$ waves in the presence of extracellular calcium [21]. This could be explained by the fact that in our cultured VSMCs, ET-1 is a weak agonist, and a number of studies have shown that both the velocity and frequency of smooth muscle $\mathrm{Ca}^{2+}$ waves increase with the level of activation and the concentration of $\mathrm{IP}_{3}$ [10,22]. The profound decrease in velocity with time is likely due to inhibition of SR refilling due to removal of $\mathrm{Ca}^{2+}$ from the bathing medium. Our findings are also in accord with a well-controlled recent study in voltage-clamped freshly isolated visceral SMCs suggesting that the observed $\mathrm{Ca}^{2+}$ waves in these cells were generated by $\mathrm{IP}_{3}$, were independent of RyRs, and were arrested by local $\mathrm{Ca}^{2+}$ buffering [21]. Interestingly, the same study also showed that in the absence of external $\mathrm{Ca}^{2+}$, the wave velocity decreased with time. Moreover, it is evident that as the depletion wave progresses, the intensity of the FRET/CFP signal at the rim of the circular 

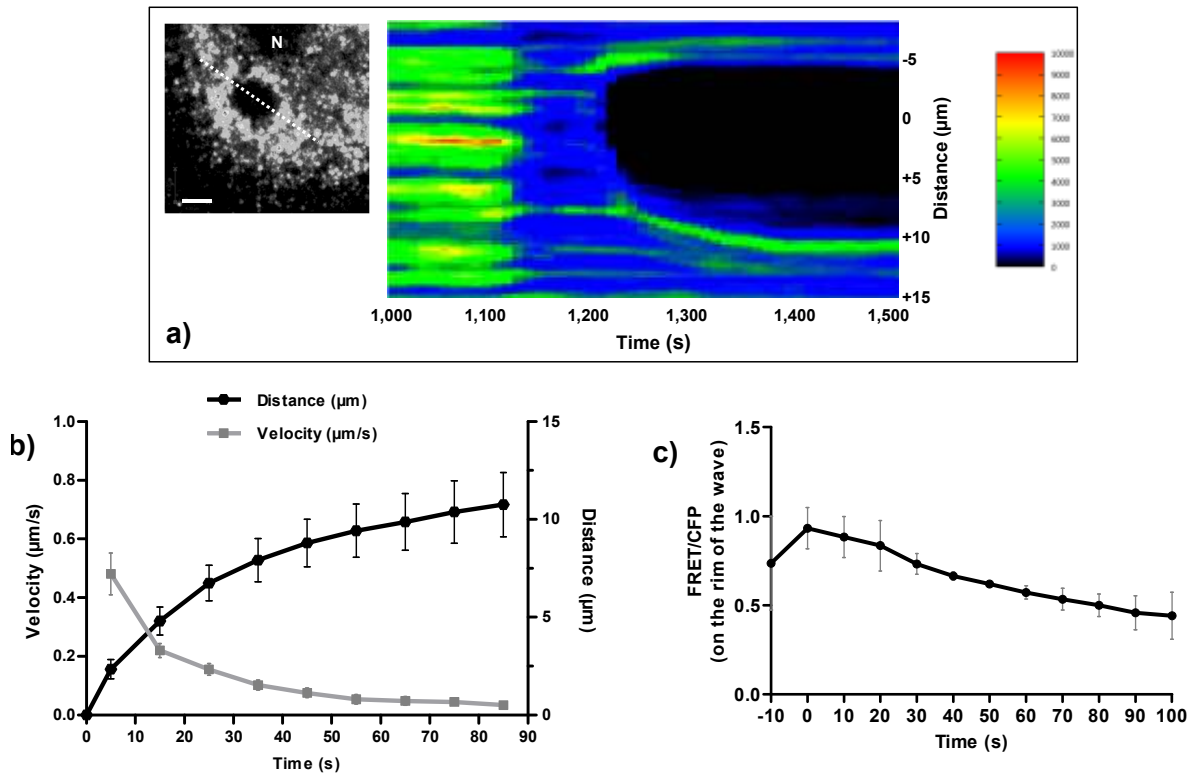

Figure 3: Dynamics of the ET-induced $\mathrm{Ca}^{2+}$ depletion wave in SMCs SR lumen. (a) Heat map generated from the time-lapse fluorescence microscopy movie $(10 \mathrm{~s}$ intervals) of rat SMCs treated with ET-1 (100 nM) in the absence of extracellular $\mathrm{Ca}^{2+}$, showing that the SR depletion wave expands in a near circular fashion in both directions. (b) Average travel distance and velocity of ET-induced depletion wave vs. time. As the distance from the point of origin increases, the wave velocity decreases asymptotically to zero. (c) Average $\mathrm{Ca}^{2+}$ signal intensity at the rim of the depletion wave also decreases as the wave expands over time ( $\mathrm{ROI}=5, \mathrm{n}=5$ cells from five independent experiments, mean \pm s.e.m., $P<0.005)$.

wave declines (Fig. 3c).

Let us consider how these new findings provide additional insight into the mechanism of excitatory $\mathrm{Ca}^{2+}$ waves. Why do we only see these waves in the absence of external $\mathrm{Ca}^{2+}$ ? And how are they initiated? Removal of external $\mathrm{Ca}^{2+}$ causes marked lowering of $\left[\mathrm{Ca}^{2+}\right]_{\mathrm{i}}$ and delays $\mathrm{SR} \mathrm{Ca}^{2+}$ release by ET-1. In our VSMC preparation, we have shown that the release is mediated by opening of $\mathrm{IP}_{3} \mathrm{R}$ (Fig. 2e). The reduced $\left[\mathrm{Ca}^{2+}\right]_{\mathrm{i}}$ lowers the rate of $\mathrm{IP}_{3}$ synthesis by phospholypase $\mathrm{C}$ (PLC), which could account for a slower increase in $\mathrm{IP}_{3}$ concentration and thus a longer latency period $[23,24]$. The latency period would be further increased because the low $\left[\mathrm{Ca}^{2+}\right]_{\mathrm{i}}$ would also raise the threshold for $\mathrm{IP}_{3}$-mediated activation. The observed transient increase in $\left[\mathrm{Ca}^{2+}\right]_{\mathrm{SR}}$ at both the origin and the rim of the depletion wave (Fig. 2c and 2d) suggests an important role for focal $\left[\mathrm{Ca}^{2+}\right]_{\mathrm{SR}}$ elevation during initiation and propagation of regenerative $\mathrm{IP}_{3} \mathrm{R}$-mediated $\mathrm{Ca}^{2+}$ release. Since in $\left[\mathrm{Ca}^{2+}\right]_{\mathrm{i}}$ oscillations, the latency period is related to the inter-spike interval [3], the smooth muscle activity may well be controlled by focal fluctuations in $\left[\mathrm{Ca}^{2+}\right]_{\mathrm{SR}}$. In figure $4 \mathrm{a}$, and in line with previous reports [25], we propose that these fluctuations are generated by a differential distribution of SR luminal $\mathrm{Ca}^{2+}$ sinks (clusters of $\mathrm{IP}_{3} \mathrm{Rs}$ ), and $\mathrm{Ca}^{2+}$ sources (SERCA). Stochastic opening of individual $\mathrm{IP}_{3} \mathrm{Rs}$ (which yield cytoplasmic $\mathrm{Ca}^{2+}$ blips) would add variability to focal $\left[\mathrm{Ca}^{2+}\right]_{\mathrm{SR}}$. Whenever a local $\left[\mathrm{Ca}^{2+}\right]_{\mathrm{SR}}$ fluctuation exceeds the threshold for regenerative opening of a $\mathrm{IP}_{3} \mathrm{R}$ cluster, sufficient $\mathrm{Ca}^{2+}$ would be released to initiate an excitatory $\mathrm{Ca}^{2+}$ wave. 

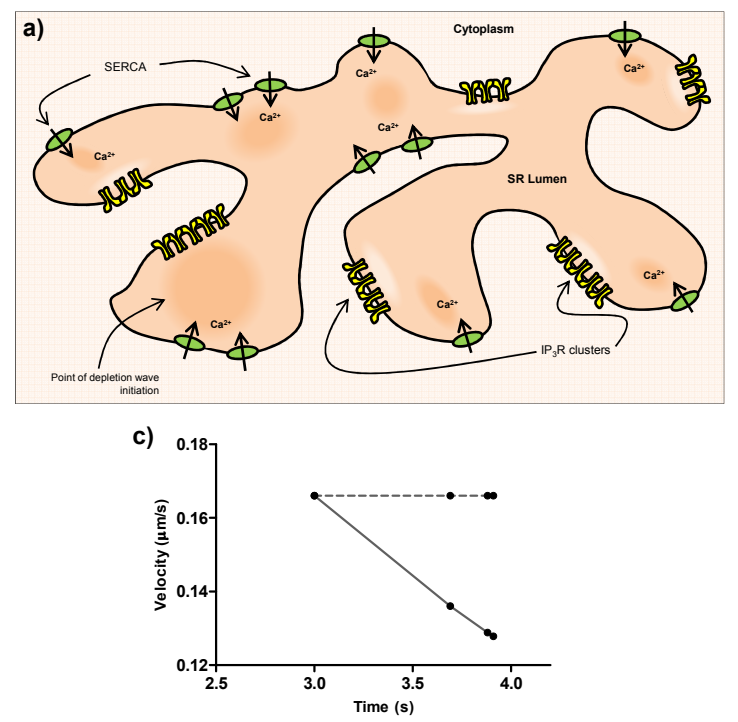

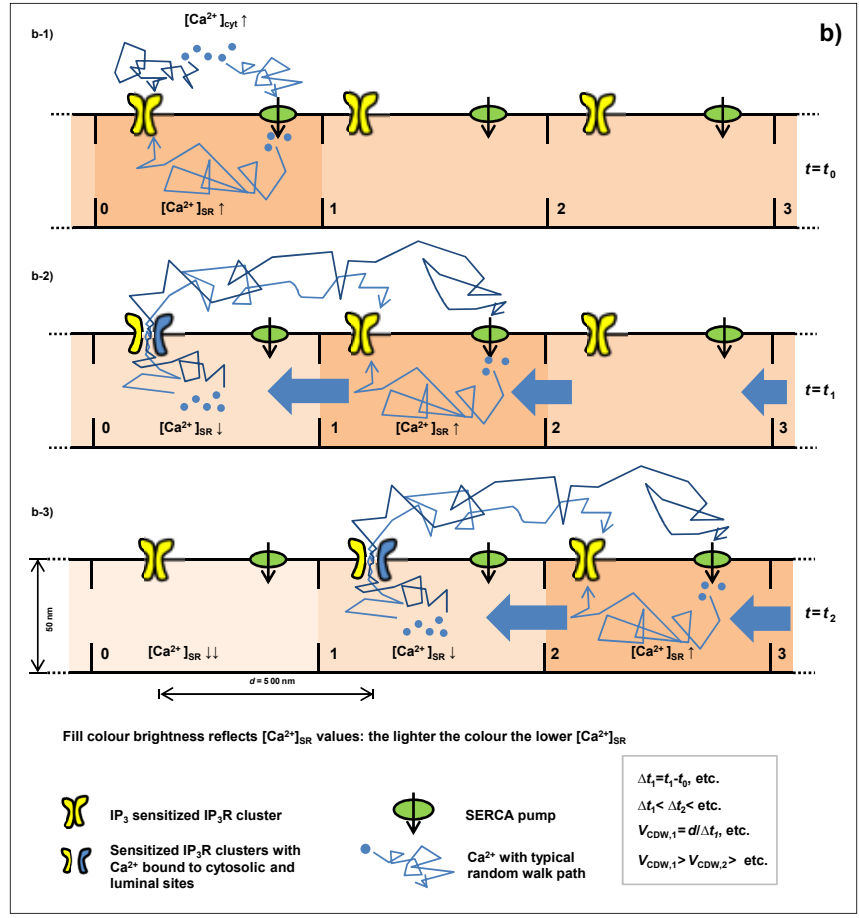

Figure 4: Simlified quantitative model for propagation of the $\mathrm{SR}^{\mathrm{Ca}^{2+}}$ depletion Wave. (a) Cartoon representation of SR network depicting differential distribution of SR luminal $\mathrm{Ca}^{2+}$ sources (SERCA) and sinks (IP $\left.{ }_{3} \mathrm{Rs}\right)$ (b) We represent a linear section of SR as a series of compartments (labeled 0, 1, 2, etc), between which $\mathrm{Ca}^{2+}$ transport is allowed. Assuming that the $\mathrm{IP}_{3}$ concentration in cytoplasm is such that the $\mathrm{IP}_{3} \mathrm{R}$ is already $\mathrm{Ca}^{2+}$-sensitized, a $\left[\mathrm{Ca}^{2+}\right]_{\mathrm{i}}$ fluctuation near the SR compartment 0 at $t=t_{0}$ raises the $\left[\mathrm{Ca}^{2+}\right]_{\mathrm{SR}}$ from a resting level to a threshold level, and thereby, causes the observed transient rise (b-1). This rise is followed by depletion of $\mathrm{Ca}^{2+}$ from compartment 0. Partial equilibration of luminal $\mathrm{Ca}^{2+}$ then takes place (blue block arrows between compartments), accompanied by an increase in $\left[\mathrm{Ca}^{2+}\right]_{\mathrm{SR}}$ in compartment 1 (b-2). Subsequently, $\left[\mathrm{Ca}^{2+}\right]_{\mathrm{SR}}$ in compartment 1 reaches a threshold value, releases (some of) its $\mathrm{Ca}^{2+}$, which goes on to partially refill its nearest neighbouring locus (compartment 2), and so on (b-3). $\mathrm{Ca}^{2+}$ release from compartment 1 can trigger release from compartment 2 , only after $\left[\mathrm{Ca}^{2+}\right]_{\mathrm{SR}}$ will have reached a critical level for $\mathrm{Ca}^{2+}$ release, which will take a time interval $\Delta t_{2}$. Due to the $\mathrm{Ca}^{2+}$ passage from compartment 2 to $1, \Delta t_{2}$ must be greater than $\Delta t_{1}$, the interval it took to raise $\left[\mathrm{Ca}^{2+}\right]_{\mathrm{SR}}$ in 1 to the release level. Successive compartment refilling times to release level will get progressively longer because of the ability to re-equilibrate $\mathrm{Ca}^{2+}$ depletions. Assuming now that the CICR sustaining $\mathrm{IP}_{3} \mathrm{R}$ clusters are on average equidistant at a length $d(500 \mathrm{~nm})$ from one another, the calcium depletion wave velocity $\left(v_{\mathrm{CDW}}\right)$, as the wave reaches successive compartments, is given by $v_{\mathrm{CDW}, 1}=d / \Delta t_{1}>v_{\mathrm{CDW}, 2}>$ etc. Under the conditions of the reported experiments, disallowing $\mathrm{Ca}^{2+}$ communication between SR compartments in this model would translate in an equal interval $\Delta t$ between the wave arrival at each compartment, and therefore in a constant wave velocity $\left(v_{\mathrm{CDW}}=\Delta t\right)$. (c) Calcium depletion wave velocity vs. time calculated from our model, considering calcium communicating (solid curve) or calcium tight (dashed) SR compartments (see Materials \& Methods for further details). 
Assuming that the SR is, more or less, evenly distributed across the cytoplasm, and that under control conditions the non-activated SR remains loaded with $\mathrm{Ca}^{2+}$ because of continual activity of SERCA, it is relatively straightforward to quantitatively simulate a typical smooth muscle $\mathrm{Ca}^{2+}$ wave of constant velocity. However, when removal of extracellular $\mathrm{Ca}^{2+}$ blocks $\mathrm{SR}$ refilling and assuming that $\mathrm{Ca}^{2+}$ is free to redistribute along the SR lumen, then opening of $\mathrm{IP}_{3} \mathrm{Rs}$ at the wave front would partially deplete the SR located just ahead of the wave (Fig. $4 b)$. Introduction of such luminal $\mathrm{Ca}^{2+}$ flux into our preliminary quantitative model indeed caused incremental slowing of the simulated $\mathrm{Ca}^{2+}$ depletion wave (Fig. 4c). This assumption is in agreement with the continuous decline in $\left[\mathrm{Ca}^{2+}\right]_{\mathrm{SR}}$ at the rim of the depletion wave (Fig. 3c).

These data collectively suggest that the elevated $\left[\mathrm{Ca}^{2+}\right]_{\mathrm{SR}}$ at the origin and at the rim of the wave plays a crucial role in wave initiation and propagation, and underscores the importance of the previous reports demonstrating that $\left[\mathrm{Ca}^{2+}\right]_{\mathrm{SR}}$ plays a stimulatory role in $\mathrm{IP}_{3} \mathrm{R}$-mediated $\mathrm{Ca}^{2+}$ release [26-28]. In fact, several reports make strong cases for the existence of a luminal excitatory $\mathrm{Ca}^{2+}$ binding site on $\mathrm{IP}_{3} \mathrm{R}$, which would provide a straightforward explanation for the stimulating effects of $\left[\mathrm{Ca}^{2+}\right]_{\mathrm{SR}}[29-32]$.

Close inspection of the SR depletion waves reported herein also provides novel insight related to sub-cellular differential $\mathrm{Ca}^{2+}$ signaling. For example, figure 5 a presents snapshots of a depletion wave, which originates very close to the nucleus. In this particular case, the shape of wave front becomes oblong instead of circular, as it fails to involve the nuclear envelope (white arrows), and proceeds in the opposite peripheral direction. The asymmetry of this particular wave is clearly demonstrated by the heat map in figure 5b. As shown, the distance traveled by the wave and its velocity also drop significantly when the wave collides with the nuclear membrane. In contrast, the pattern of two neighbouring SR depletion waves colliding with each other is clearly different from the case when a depletion wave encounters the nuclear or plasma membrane (Fig. 5c). A line scan of the $\mathrm{Ca}^{2+}$ signal through the point of origin and through the border between neighbouring waves ( $a$ and b) shows that the depleted areas between two adjoining depletion waves coalesce (white arrow) without a dividing line of $\mathrm{Ca}^{2+}$ loaded $\mathrm{SR}$ (red arrows).

Our observation of smooth muscle $\mathrm{SR} \mathrm{Ca}^{2+}$ depletion waves demonstrates that during agonist stimulation of vascular smooth muscle cells, fluctuations in $\left[\mathrm{Ca}^{2+}\right]_{\mathrm{SR}}$ do not necessarily mirror changes in $\left[\mathrm{Ca}^{2+}\right]_{\mathrm{i}}$, but can provide important novel mechanistic insight into complex cellular $\mathrm{Ca}^{2+}$ signalling. The transient elevations of $\left[\mathrm{Ca}^{2+}\right]_{\mathrm{SR}}$ at the site of wave initiation and at the wave front, directly demonstrate local interactions between adjacent SR sites, which could be readily influenced by other organelles, such as mitochondria and lysosomes in shaping the smooth muscle $\mathrm{Ca}^{2+}$ signalling [33-35]. Furthermore, the indication that the SR depletion waves choose a preferred path by avoiding the nucleus may have physiological implications and relate to our earlier suggestion that vascular dysfunction in aging and disease could be related to loss of vascular smooth muscle $\mathrm{Ca}^{2+}$ waves [36]. 

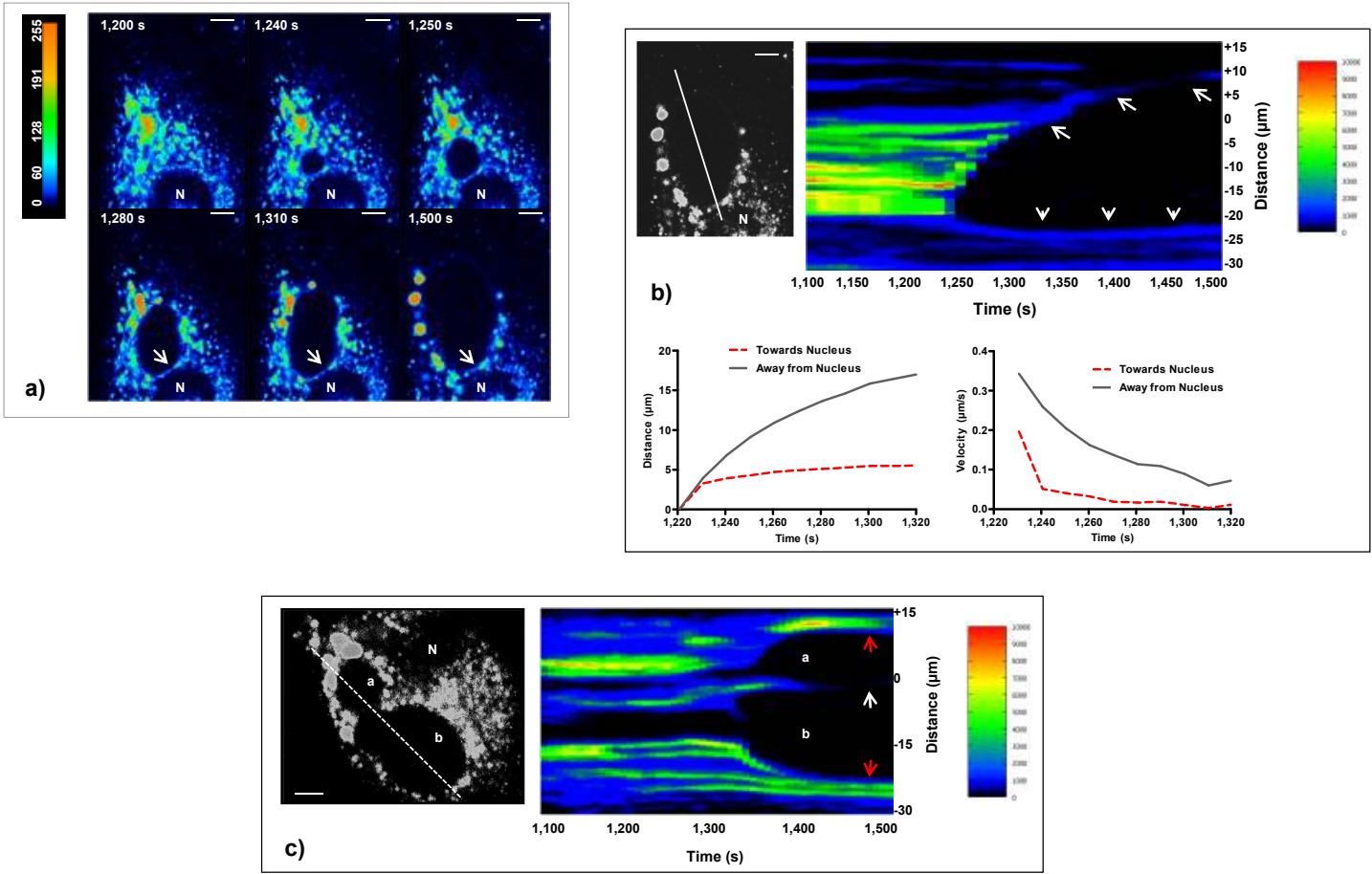

Figure 5: Arrestment of ET-induced depletion wave at the border of nuclear envelope and plasma membrane in cultured SMCs. (a) Pseudo-colour snapshots (FRET channel) of time-lapse movie (10 s intervals) of cultured SMCs transfected with D1SR and treated with ET-1 (100 nM) in the absence of extracellular $\mathrm{Ca}^{2+}$. The depletion wave is arrested at the nuclear envelope (white arrows), but progresses in the opposite direction (scale bars, $5 \mu \mathrm{m}$; N, nucleus). (b) Line scan of $\mathrm{Ca}^{2+}$ signal through the point of origin of the depletion wave and heat map of the arrested wave. As shown, the depletion wave is unable to proceed through nuclear region and is arrested at the nuclear border creating an almost straight line of calcium-rich areas (white arrowheads), while progressing in the opposite direction (white arrows) (scale bars, $5 \mu \mathrm{m}$ ). Traces compare the measurement of distance travelled and velocity of the wave over time toward and away from the nuclear envelope from the point of origin. (c) Line scan of $\mathrm{Ca}^{2+}$ signal through the point of origin of two neighbouring depletion waves ( $a$ and $b$ ) and heat map of the colliding waves accentuate that the bright rim between two neighbouring waves disappears over time (white arrows), while the rims facing plasma membrane persist (red arrows) (N, nucleus, scale bars, $5 \mu \mathrm{m}$ ). 


\section{Materials \& Methods}

\section{Cell Culture}

Rat aortic smooth muscle cells were cultured in Matrigel-coated (BD Sciences; ON, Canada) $35 \mathrm{~mm}$ glass bottom culture dishes (MatTek Co., MA, USA) and grown in Dulbecco's modified Eagle's medium supplemented with $10 \%$ heat-inactivated newborn calf serum, Penicillin $\mathrm{G}\left(100 \mu \mathrm{g} \cdot \mathrm{mL}^{-1}\right)$, and streptomycin $\left(100 \mu \mathrm{g} \cdot \mathrm{mL}^{-1}\right)$ (Invitrogen, ON, Canada)) at $37^{\circ} \mathrm{C}$, in a humidified incubator in $5 \% \mathrm{CO}_{2}$. To assure the consistency of results, passage 20-23 of SMCs was used for all experiments.

\section{Buffer \& Reagents}

HEPES-PSS containing (in mmol.mL ${ }^{-1}$ ) $\mathrm{NaCl} 140$, glucose 10, $\mathrm{KCl} 5$, HEPES $5, \mathrm{CaCl}_{2} 1.5$ and $\mathrm{MgCl}_{2} 1$ ( $\mathrm{pH}$ 7.4) was used for all calcium measurements and confocal microscopy. The nominal zero- $\mathrm{Ca}^{2+}$ PSS was prepared in the same way as normal PSS without the addition of calcium. Endothelin-1 (ET-1) was obtained from Sigma-Aldrich (ON, Canada). Thapsigargin $(\mathrm{Tg})$, a cell permeable inhibitor of sarcoplasmic reticular $\mathrm{Ca}^{2+}$-ATPase (SERCA), and xestospongin $\mathrm{C}$ (Xes-C), a potent membrane-permeable blocker of $\mathrm{IP}_{3}$-mediated $\mathrm{Ca}^{2+}$ release, were purchased from EMD Chemicals (NJ, USA). Sodium ortovanadate, a selective inhibitor of secretory pathway $\mathrm{Ca}^{2+}$ ATPase (SPCA) pumps, was purchased from SigmaAldrich. Stock solutions of ET-1 and Tg were prepared in dimethyl sulfoxide (DMSO). For all experiments, vehicle-treated $(0 \mu \mathrm{M})$ groups were incubated with equal volume of DMSO, respectively (the maximum volume of solvents used with the highest concentration of drugs). Further dilutions of reagents were made in zero- $\mathrm{Ca}^{2+}$ PSS buffer. The calcium dye Fluo4AM, ER-Tracker, Mito-Tracker Green FM, and CellLight ${ }^{\mathrm{TM}}$ Golgi-GFP BacMam 2.0 were all purchased from Invitrogen.

\section{Cytoplasmic calcium measurement}

For in vitro measurement of cytoplasmic calcium signals, sub-confluent SMCs were grown on Matrigel-coated 35-mm glass-bottom culture dishes $48 \mathrm{~h}$ prior to each experiment. Cells were then loaded with Fluo-4AM for $1 \mathrm{~h}$ at $37^{\circ} \mathrm{C}$, followed by a $15-20$-minute wash in HEPES-buffered physiological saline solution. Images were acquired using the Spinning Disk Confocal system (Leica DM16000 inverted microscope) with a 63X oil-immersion objective and equipped with the Improvision Volocity imaging system (Perkin-Elmer). All parameters (laser intensity, gain, etc.) were maintained constant during the experiment. The cell culture was illuminated using an Argon-Krypton laser (488nm) and a high-gain photomultiplier tube collected the emission (505-550 nm). The customized Hamamatsu 9100-02 electron multiplier CCD camera delivers 1000 X 1000, imaged to Shannon-Nyquist specifications for the $63 \mathrm{X}$ objectives, providing a larger field of view. The representative fluorescence traces shown reflect the averaged fluorescence signals from 15 regions of interest (ROIs) in each cell. The measured changes in Fluo-4AM fluorescence level are proportional to the relative changes in cytoplasmic calcium $\left(\left[\mathrm{Ca}^{2+}\right]_{\mathrm{i}}\right)$. The confocal images were analyzed off-line with the Improvision Volocity software (Perkin-Elmer). Fluorescence traces were extracted from the 
movies and were normalized to initial fluorescence values. Live imaging of SR, mitochondrial and Golgi apparatus in culture SMCs Sub-confluent SMCs were grown on Matrigel-coated 35-mm glass-bottom culture dishes, and were then loaded with different probes as follow. For $\mathrm{SR} / \mathrm{ER}$ and mitochondria staining, cells were incubated for 15 minutes in Hanks Balanced Salt Solution with calcium and magnesium containing $1 \mu \mathrm{M}$ ER-Tracker ${ }^{\mathrm{TM}}$ Red Dye and $100 \mathrm{nM}$ MitoTracker Green FM at $37^{\circ} \mathrm{C}$. For Golgi staining, cells were incubated with $1 \mathrm{~mL}$ culture medium containing $1 \mu \mathrm{L}$ of Golgi-GFP BacMam reagent $\left(1 \times 10^{6}\right.$ particles $)$ overnight prior to loading with ER-Tracker for 15 minutes. The staining solutions were replaced by HEPES-buffered physiological saline solution, and cells were viewed with the Spinning Disk Confocal system (Leica DM16000 inverted microscope) with a 63X oil-immersion objective (ER-Tracker: $587 \mathrm{~nm}$ excitation/615 nm emission, Mito-Tracker Green FM and Golgi-GFP: $488 \mathrm{~nm}$ excitation/516 $\mathrm{nm}$ emission).

\section{Live imaging of SR, mitochondrial and Golgi apparatus in culture SMCs}

Sub-confluent SMCs were grown on Matrigel-coated 35-mm glass-bottom culture dishes, and were then loaded with different probes as follow. For SR/ER and mitochondria staining, cells were incubated for 15 minutes in Hank's Balanced Salt Solution with calcium and magnesium containing $1 \mu \mathrm{M}$ ER-Tracker ${ }^{\mathrm{TM}}$ Red Dye and $100 \mathrm{nM}$ MitoTracker Green FM at $37^{\circ} \mathrm{C}$. For Golgi staining, cells were incubated with $1 \mathrm{~mL}$ culture medium containing $1 \mu \mathrm{L}$ of Golgi-GFP BacMam reagent $\left(1 \times 10^{6}\right.$ particles $)$ overnight prior to loading with ER-Tracker for 15 minutes. The staining solutions were replaced by HEPES-buffered physiological saline solution, and cells were viewed with the Spinning Disk Confocal system (Leica DM16000 inverted microscope) with a $63 \mathrm{X}$ oil-immersion objective (ER-Tracker: $587 \mathrm{~nm}$ excitation/615 nm emission, Mito-Tracker Green FM and Golgi-GFP: 488 nm excitation/516 nm emission).

\section{Transient Transfection with D1SR Construct}

Calcium signal within the SR was measured with a FRET-based probe D1SR $\left(K_{d}=60-\right.$ $180 \mu \mathrm{mol} / \mathrm{L})$, a modified version of the D1ER construct originally designed by Dr. Roger Tsien (University of California, San Diego, USA). The D1SR construct consists of a truncated enhanced CFP and citrine fluorescent protein that are joined by a linker and hinge containing modified calmodulin and M13 sequences with a biphasic $\mathrm{Ca}^{2+}$ response measured in vitro with $K_{d}$ 's $\left[\mathrm{Ca}^{2+}\right]$ of $\sim 0.6 \mu \mathrm{M}$ and $\sim 60 \mu \mathrm{M}$. The CaM-M13 modifications prevent M13 from binding endogenous calmodulin. SR retention is achieved by calcequestrin and KDEL retention sequences on the 5' end of CFP and 3' end of citrine, respectively. SMCs were transfected with adenoviral D1SR constructs at a multiplicity of infection of 100 (MOI=100). Following overnight incubation at $37^{\circ} \mathrm{C}$, cells were replenished with fresh medium. Fluorescence microscopy was used to assess transfection efficiency and cellular morphology at $48 \mathrm{~h}$ post transfection. For all experiments, a transfection efficiency of 80-90\% was achieved. 


\section{SR luminal calcium measurement}

Ratiometric FRET images were acquired with a 63X oil-immersion objective (Leica DM16000 inverted microscope) and a cooled Hamamatsu 9100-02 electron multiplier CCD camera. Cells were excited at $440 \mathrm{~nm}$, and $513 \mathrm{~nm}$; and imaged using $488 \mathrm{~nm} / 535 \mathrm{~nm}$ (for donor and FRET channels) and $535 \mathrm{~nm}$ (for acceptor channel) band-pass filters. Three images were taken at each time point (CFP, FRET, YFP). The direct YFP image (513 nm excitation, 535 $\mathrm{nm}$ emission) was simultaneously recorded to monitor photobleaching. We set the intensity of light at 30\% transmitted light, and excitation exposure times at $150 \mathrm{~ms}$ with images acquired every $10 \mathrm{~s}$. To calculate the FRET ratio, the values for FRET measurements were divided by values for CFP measurements. Changes in FRET/CFP ratio is a representative of changes in SR luminal calcium.

\section{Image analysis}

All data used for calcium traces were analyzed by Improvision Volocity software, using builtin regions-of-interest (ROI) function to select the areas of interest that is at least 30 pixels long in length and width. For traces involving specific sites, shape and size of ROI were adjusted to avoid artefacts and saturated areas. For distance and velocity analysis, one ROI was selected per frame (time point) based on the movement of the calcium wave. The resulting data were formatted on Microsoft Excel 2007, and analyzed by GraphPad Prism 5.0. Pseudo-colour visualization was performed by ImageJ, using customized lookup tables to assign colour for each pixel intensity values. Line scan was performed first by analyzing pixel intensity of a series of small ROIs (1-3 pixels in length per ROI) along a line using a customized Python script to output change in intensity over time along the line. The script was confirmed to output same values when analyzing same ROI on Volocity (data not shown). The resulting values were then graphed using Gnuplot 4.4 with custom lookup table well.

\section{Quantitative Modeling}

The results produced by the quantitative model illustrated in Figure 4 were obtained using the data in table 1.

The proposed quantitative model for propagation of the depletion wave is based on previous suggested models arguing for 1 ) a continuous SR lumen in regards to $\mathrm{Ca}^{2+}$ transport, 2) the appearance of functionally segregated compartments in the SR of SMCs, and 3) the existence of a luminal $\mathrm{Ca}^{2+}$ binding site of the $\mathrm{IP}_{3} \mathrm{R}[30,31,40]$. The model assumes that, before any $\mathrm{Ca}^{2+}$ depletion wave $(\mathrm{CDW})$ event, the $\left[\mathrm{Ca}^{2+}\right]_{\mathrm{SR}}$ is at a normal level $\left(C_{\mathrm{SR}}\right)$ and that it needs to reach a critical level $\left(C_{\mathrm{SR}}^{*}\right)$ for release. Then, for example, following the depletion of a given SR compartment, for a nearest neighbouring compartment to release, its $\left[\mathrm{Ca}^{2+}\right]_{\mathrm{SR}}$ needs to change by $C_{\mathrm{SR}}^{*}-C_{\mathrm{SR}}=40 \mu \mathrm{M}$, which translates to about $300 \mathrm{Ca}^{2+}$. For the sake of order-of-magnitude calculations, let us say that 3 SERCA operating at 100 $\mathrm{s}^{-1}$ would take a time interval $\Delta t_{1}=3 \mathrm{~s}$ to cause a $40 \mu \mathrm{M}\left[\mathrm{Ca}^{2+}\right]_{\mathrm{SR}}$ change (in reality, there are likely many more SERCA working at considerably lower turnover rates). We calculate the depletion wave velocity, $v_{\mathrm{CDW}}$, as the inter-cluster distance, $d$, over the time, $\Delta t$, taken 


\begin{tabular}{l|l|l}
\hline \hline SR lumen cross-section & $50 \mathrm{~nm} \times 50 \mathrm{~nm}$ & {$[37]$} \\
$\mathrm{IP}_{3} \mathrm{R}$ cluster-cluster distance & $500 \mathrm{~nm}$ & {$[38]$} \\
SR compartment volume & $50 \mathrm{~nm} \times 50 \mathrm{~nm} \times 500 \mathrm{~nm}$ & \\
SERCA refill rate & $100 \mathrm{~s}^{-1}$ & {$[39]$} \\
Normal $\left[\mathrm{Ca}^{2+}\right]_{\mathrm{SR}}, C_{\mathrm{SR}}$ & $60 \mu \mathrm{M}$ & {$[16]$} \\
$\mathrm{Critical}\left[\mathrm{Ca}^{2+}\right]_{\mathrm{SR}}, C_{\mathrm{SR}}^{*}$ & $100 \mu \mathrm{M}$ & {$[16]$} \\
$\mathrm{Ca}^{2+}$ shift from 1 to 0 & $20 \%$ & \\
$\mathrm{Ca}^{2+}$ shift from 2 & $5 \%$ & \\
$\mathrm{Ca}^{2+}$ shift from 3 & $1 \%$ & \\
\hline \hline
\end{tabular}

Table 1: Summary of data used in the model.

to raise the $\left[\mathrm{Ca}^{2+}\right]_{\mathrm{SR}}$ in the new compartment from normal to critical: $v_{\mathrm{CDW}}=d / \Delta t$. Using the data in the table and referring to Figure 4b, for one depletion "step" in our model, $v_{\mathrm{CDW}, 1}=d / \Delta t_{1}=0.166 \mu \mathrm{m} / \mathrm{s}$. If we now assume that SR compartments 2,3 , etc lose part of their $\mathrm{Ca}^{2+}$ toward the depleted neighbouring compartments as indicated in the table (these percentages are arbitrarily chosen), their "normal" $\left[\mathrm{Ca}^{2+}\right]_{\mathrm{SR}}$ will be $C_{\mathrm{SR}, 2}, C_{\mathrm{SR}, 3}$, etc., lower than $C_{\mathrm{SR}}$. The same line of reasoning then suggests that, for compartment 2 to release, its $\left[\mathrm{Ca}^{2+}\right]_{\mathrm{SR}}$ needs to change by $C_{\mathrm{SR}}^{*}-C_{\mathrm{SR}}>40 \mu \mathrm{M}$, which will take a time $\Delta t_{2}>\Delta t_{1}$ and yield a velocity for the second step as $v_{\mathrm{CDW}, 2}=d / \Delta t_{2}<v_{\mathrm{CDW}, 1}$.

\section{Acknowledgements}

We thank Dr. Wayne Chen at Libin Cardiovascular Institute of Alberta, University of Alberta for his assistance with the design of the D1SR construct. We also thank Dr. Kevin Hodgson at University of British Columbia Bio-Imaging Facility for his technical assistance with confocal microscopy.

\section{Author Contributions}

M.E. and C.v.B. designed the experiments. M.E. and Y.C. carried out the experiments. M.E., Y.C., A.Y.T., and J.G.H. performed data analyses. N.F. and C.v.B. developed the quantitative model.

\section{References}

[1] M. Iino, H. Kasai, and T. Yamazawa. Visualization of neural control of intracellular ca2+ concentration in single vascular smooth muscle cells in situ. EMBO J, 13(21):50265031, Nov 1994. 
[2] Christian Aalkjaer and Holger Nilsson. Vasomotion: cellular background for the oscillator and for the synchronization of smooth muscle cells. Br J Pharmacol, 144(5):605-616, Mar 2005.

[3] M. J. Berridge. Cytoplasmic calcium oscillations: a two pool model. Cell Calcium, 12(2-3):63-72, 1991.

[4] L. A. Blatter and W. G. Wier. Agonist-induced $[$ ca2+]i waves and ca $(2+)$-induced ca2+ release in mammalian vascular smooth muscle cells. Am J Physiol, 263(2 Pt 2):H576H586, Aug 1992.

[5] P. Nicotera and S. Orrenius. The role of calcium in apoptosis. Cell Calcium, 23(23):173-180, 1998.

[6] B. Kumar, K. Dreja, S. S. Shah, A. Cheong, S-Z. Xu, P. Sukumar, J. Naylor, A. Forte, M. Cipollaro, D. McHugh, P. A. Kingston, A. M. Heagerty, C. M. Munsch, A. Bergdahl, A. Hultgrdh-Nilsson, M. F. Gomez, K. E. Porter, P. Hellstrand, and D. J. Beech. Upregulated trpc1 channel in vascular injury in vivo and its role in human neointimal hyperplasia. Circ Res, 98(4):557-563, Mar 2006.

[7] Cornelis van Breemen, Damon Poburko, and Elena B Okon. Trp proteins: a new dimension in the treatment of occlusive vascular disease. Circ Res, 98(4):446-447, Mar 2006.

[8] M. J. Berridge, P. Lipp, and M. D. Bootman. The versatility and universality of calcium signalling. Nat Rev Mol Cell Biol, 1(1):11-21, Oct 2000.

[9] E. Carafoli, L. Santella, D. Branca, and M. Brini. Generation, control, and processing of cellular calcium signals. Crit Rev Biochem Mol Biol, 36(2):107-260, Apr 2001.

[10] Masamitsu Iino. Spatiotemporal dynamics of ca2+ signaling and its physiological roles. Proc Jpn Acad Ser B Phys Biol Sci, 86(3):244-256, 2010.

[11] C. B. Neylon, J. Hoyland, W. T. Mason, and R. F. Irvine. Spatial dynamics of intracellular calcium in agonist-stimulated vascular smooth muscle cells. Am J Physiol, 259(4 Pt 1):C675-C686, Oct 1990.

[12] M. J. Berridge and R. F. Irvine. Inositol phosphates and cell signalling. Nature, 341(6239):197-205, Sep 1989.

[13] M. Iino. Biphasic ca2+ dependence of inositol 1,4,5-trisphosphate-induced ca release in smooth muscle cells of the guinea pig taenia caeci. J Gen Physiol, 95(6):1103-1122, Jun 1990.

[14] A. Miyawaki, J. Llopis, R. Heim, J. M. McCaffery, J. A. Adams, M. Ikura, and R. Y. Tsien. Fluorescent indicators for ca2+ based on green fluorescent proteins and calmodulin. Nature, 388(6645):882-887, Aug 1997. 
[15] Amy E Palmer and Roger Y Tsien. Measuring calcium signaling using genetically targetable fluorescent indicators. Nat Protoc, 1(3):1057-1065, 2006.

[16] Damon Poburko, Chiu-Hsiang Liao, Cornelis van Breemen, and Nicolas Demaurex. Mitochondrial regulation of sarcoplasmic reticulum ca2 + content in vascular smooth muscle cells. Circ Res, 104(1):104-112, Jan 2009.

[17] Cheng-Han Lee, Damon Poburko, Kuo-Hsing Kuo, Chun Seow, and Cornelis van Breemen. Relationship between the sarcoplasmic reticulum and the plasma membrane. Novartis Found Symp, 246:26-41; discussion 41-7, 48-51, 2002.

[18] P. A. Leijten and C. van Breemen. The relationship between noradrenaline-induced contraction and 45ca efflux stimulation in rabbit mesenteric artery. $\mathrm{Br} J$ Pharmacol, 89(4):739-747, Dec 1986.

[19] John G McCarron, Susan Chalmers, and Thomas C Muir. "quantal" ca2+ release at the cytoplasmic aspect of the ins $(1,4,5) \mathrm{p} 3 \mathrm{r}$ channel in smooth muscle. J Cell Sci, 121(Pt 1):86-98, Jan 2008.

[20] O. Vallot, L. Combettes, P. Jourdon, J. Inamo, I. Marty, M. Claret, and A. M. Lompr. Intracellular ca $(2+)$ handling in vascular smooth muscle cells is affected by proliferation. Arterioscler Thromb Vasc Biol, 20(5):1225-1235, May 2000.

[21] John G McCarron, Susan Chalmers, Debbi MacMillan, and Marnie L Olson. Agonistevoked ca(2+) wave progression requires ca(2+) and ip(3). J Cell Physiol, 224(2):334344, Aug 2010.

[22] Cheng-Han Lee, Roshanak Rahimian, Tania Szado, Jasmin Sandhu, Damon Poburko, Tasniem Behra, Lally Chan, and Cornelis van Breemen. Sequential opening of ip(3)sensitive $\mathrm{ca}(2+)$ channels and soc during alpha-adrenergic activation of rabbit vena cava. Am J Physiol Heart Circ Physiol, 282(5):H1768-H1777, May 2002.

[23] T. Meyer and L. Stryer. Molecular model for receptor-stimulated calcium spiking. Proc Natl Acad Sci U S A, 85(14):5051-5055, Jul 1988.

[24] T. Meyer and L. Stryer. Calcium spiking. Annu Rev Biophys Biophys Chem, 20:153-174, 1991.

[25] John S Fitzpatrick, Anna M Hagenston, Daniel N Hertle, Keith E Gipson, Lisa BertettoD'Angelo, and Mark F Yeckel. Inositol-1,4,5-trisphosphate receptor-mediated ca2+ waves in pyramidal neuron dendrites propagate through hot spots and cold spots. $J$ Physiol, 587(Pt 7):1439-1459, Apr 2009.

[26] L. Missiaen, C. W. Taylor, and M. J. Berridge. Luminal ca2+ promoting spontaneous ca2+ release from inositol trisphosphate-sensitive stores in rat hepatocytes. J Physiol, 455:623-640, Sep 1992. 
[27] L. Missiaen, H. De Smedt, G. Droogmans, and R. Casteels. Ca2+ release induced by inositol 1,4,5-trisphosphate is a steady-state phenomenon controlled by luminal ca2+ in permeabilized cells. Nature, 357(6379):599-602, Jun 1992.

[28] Anatoly Shmygol and Susan Wray. Modulation of agonist-induced ca2+ release by sr ca2 + load: direct sr and cytosolic ca2+ measurements in rat uterine myocytes. Cell Calcium, 37(3):215-223, Mar 2005.

[29] L. Missiaen, C. W. Taylor, and M. J. Berridge. Spontaneous calcium release from inositol trisphosphate-sensitive calcium stores. Nature, 352(6332):241-244, Jul 1991.

[30] I. Sienaert, H. De Smedt, J. B. Parys, L. Missiaen, S. Vanlingen, H. Sipma, and R. Casteels. Characterization of a cytosolic and a luminal ca2+ binding site in the type i inositol 1,4,5-trisphosphate receptor. J Biol Chem, 271(43):27005-27012, Oct 1996.

[31] L. Missiaen, H. De Smedt, J. B. Parys, I. Sienaert, S. Vanlingen, and R. Casteels. Effects of luminal ca2+ on inositol trisphosphate-induced ca2+ release: facts or artifacts? Cell Calcium, 19(1):91-93, Jan 1996.

[32] Colin W Taylor and Stephen C Tovey. Ip(3) receptors: toward understanding their activation. Cold Spring Harb Perspect Biol, 2(12):a004010, Dec 2010.

[33] Paola Pizzo and Tullio Pozzan. Mitochondria-endoplasmic reticulum choreography: structure and signaling dynamics. Trends Cell Biol, 17(10):511-517, Oct 2007.

[34] Fan Zhang, Min Xia, and Pin-Lan Li. Lysosome-dependent ca $(2+)$ release response to fas activation in coronary arterial myocytes through naadp: evidence from cd38 gene knockouts. Am J Physiol Cell Physiol, 298(5):C1209-C1216, May 2010.

[35] Nicholas P Kinnear, Christopher N Wyatt, Jill H Clark, Peter J Calcraft, Sidney Fleischer, Loice H Jeyakumar, Graeme F Nixon, and A. Mark Evans. Lysosomes co-localize with ryanodine receptor subtype 3 to form a trigger zone for calcium signalling by naadp in rat pulmonary arterial smooth muscle. Cell Calcium, 44(2):190-201, Aug 2008.

[36] Jiazhen Minnie Dai, Harley Syyong, Jorge Navarro-Dorado, Santiago Redondo, Mauricio Alonso, Cornelis van Breemen, and Teresa Tejerina. A comparative study of alphaadrenergic receptor mediated $\mathrm{ca}(2+)$ signals and contraction in intact human and mouse vascular smooth muscle. Eur J Pharmacol, 629(1-3):82-88, Mar 2010.

[37] Nicola Fameli, Cornelis van Breemen, and Kuo-Hsing Kuo. A quantitative model for linking na+/ca2+ exchanger to serca during refilling of the sarcoplasmic reticulum to sustain $[\mathrm{ca} 2+]$ oscillations in vascular smooth muscle. Cell Calcium, 42(6):565-575, Dec 2007.

[38] J. Kevin Foskett, Carl White, King-Ho Cheung, and Don-On Daniel Mak. Inositol trisphosphate receptor ca2+ release channels. Physiol Rev, 87(2):593-658, Apr 2007.

[39] Peter Läuger. Electrogenic Ion Pumps. Sinauer Associates, Sunderland, Mass., USA, 1991. 
[40] Richard D Rainbow, Debbi Macmillan, and John G McCarron. The sarcoplasmic reticulum ca2+ store arrangement in vascular smooth muscle. Cell Calcium, 46(5-6):313-322, 2009. 
a)

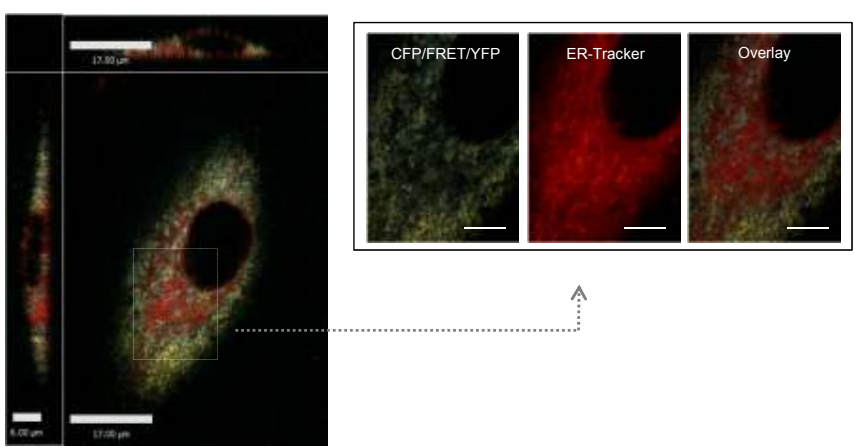

b)

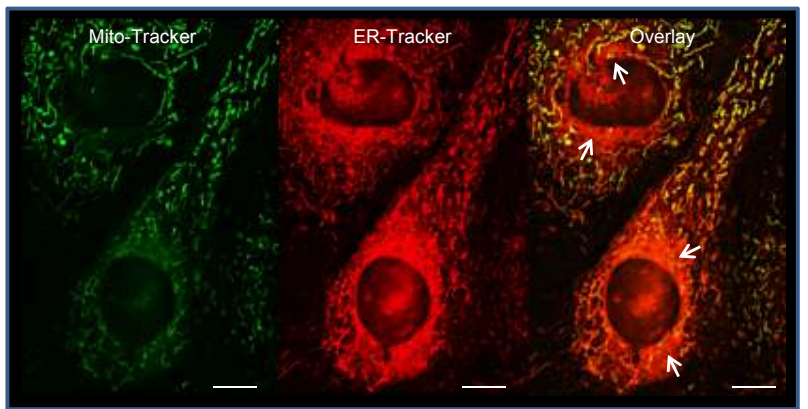

c)

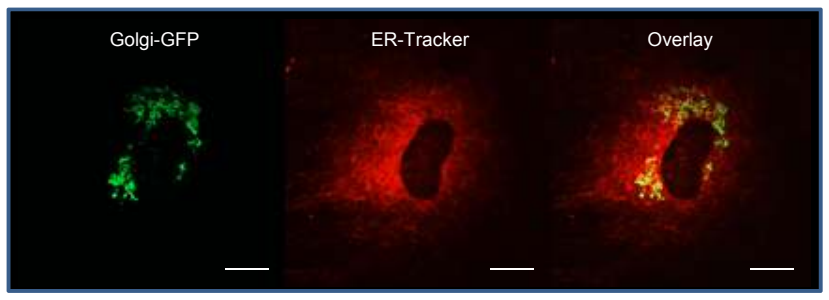

Figure 6: Distribution of SR luminal network, mitochondria, and Golgi apparatus in cultured rat SMCs. (a) Representative images of D1SR distribution in cultured rat SMCs. At 48 h post-transfection with D1SR construct, cells were loaded with the ER-Tracker ${ }^{\mathrm{TM}}$ Red dye $(1 \mathrm{i} \mu \mathrm{M})$ in HBSS $/ \mathrm{Ca}^{2+} / \mathrm{Mg}^{2+}$ buffer at $37^{\circ} \mathrm{C}$ for 15 minutes. A merged Fluorescence 3-D image of D1SR using CFP (440 nm excitation/488 nm emission), FRET (440 nm excitation/535 nm emission), and YFP (514 nm excitation/535 nm emission) band-pass filters, and ER-Tracker ${ }^{\mathrm{TM}}$ Red (587 nm excitation/615 nM emission) are shown. As shown, the majority of area shows overlay of D1SR and SR lumen tracker. The zoomed-in images highlight areas of SR lumen negative for D1SR signal, but positive for ER-Tracker. In all experiments, such areas were excluded from data analyses (scale bars, $10 \mu \mathrm{m}$ unless indicated otherwise). (b) Rat SMCs were loaded with ER-Tracker ${ }^{\mathrm{TM}}$ Red dye $(1 \mu \mathrm{M})$ and MitoTracker Green FM $(250 \mathrm{nM})$ in HBSS $/ \mathrm{Ca}^{2+} / \mathrm{Mg}^{2+}$ buffer at $37^{\circ} \mathrm{C}$ for 15 minutes. Fluorescence images of ER-Tracker ${ }^{\mathrm{TM}}$ Red (587 nm excitation/615 nM emission) and MitoTracker (488 nm excitation/520 nm emission). The white arrows indicated small peri-nuclear areas of SR that are negative for MitoTracker (scale bar, $10 \mu \mathrm{m}$ ). (c) SMCs are loaded with GolgiGFP CellLight $\AA$ solution for 16 hours at $37^{\circ} \mathrm{C}$ prior to loading with ER-Tracker ${ }^{\mathrm{TM}}$ Red dye for 15 minutes. Fluorescence images of ER-Tracker ${ }^{\mathrm{TM}}$ Red (587 nm excitation/615 nM emission) and Golgi-GFP (488 nm excitation/520 nm emission)(scale bars, $10 \mu \mathrm{m}$ ) are shown. 


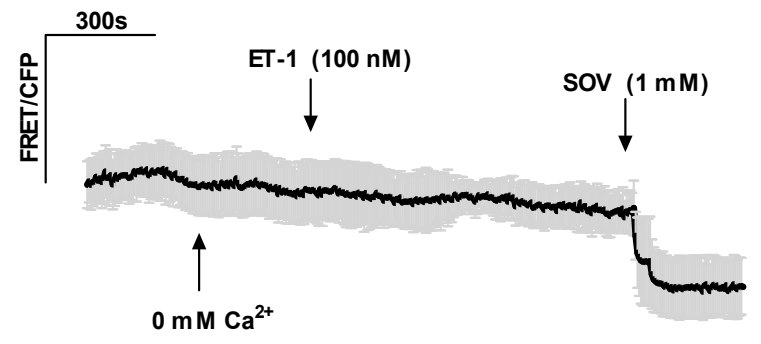

Figure 7: Elimination of $\mathrm{Ca}^{2+}$ cycling through SR luminal network by sodium orthovanadate (SOV) in cultured rat SMCs. In the absence of extracellular $\mathrm{Ca}^{2+}$ and presence of $100 \mathrm{nM}$ ET-1, SOV (1 mM) caused a significant drop in SR luminal $\mathrm{Ca}^{2+}$ and almost completely emptied the SR ( $\mathrm{n}=4$ cells from 4 independent experiments, mean \pm s.e.m., $P<0.005)$. 